\title{
HCV treatment in 2020: How to translate highly effective therapies into elimination strategies
}

\author{
(D) Omar Alshuwaykh, (D) Paul Y Kwo \\ Division of Hepatology, Department of Gastroenterology, Stanford University School of Medicine, California, USA
}

\begin{abstract}
The hepatitis $\mathrm{C}$ virus (HCV) is among the most common blood-borne infections worldwide and a major cause of cirrhosis and hepatocellular carcinoma. HCV was first identified in 1989. The current use of direct-acting antiviral agents (DAAs) to cure HCV reflects rapid diagnostic and therapeutic advances in a short period of time that is seen in few diseases. Both the cost and access to DAAs have improved since the introduction of these therapies in 2014. While HCV is very easy to treat, it will be difficult to eliminate worldwide. The tools exist to create strategies to treat and eliminate $\mathrm{HCV}$ as a public health threat; however, elimination of $\mathrm{HCV}$ will involve improving access to diagnostic testing for HCV with confirmation of active infection. Models of care will need to be revised from centralized, specialized care to decentralized, point-of-care treatment for HCV patients. These models should include clinics that care for populations with a high prevalence of $\mathrm{HCV}$, such as those treating intravenous drug users, needle exchange services, community health centers, and prisons, in addition to primary care clinics. These care pathways are feasible because of the simplicity of pangenotypic therapies for HCV that require minimal monitoring. Many countries and regions of the world have embarked on programs with the goal of achieving the World Health Organization target of elimination by 2030 . Best practices in HCV elimination should be shared globally.
\end{abstract}

Keywords: Hepatitis C; direct acting antiviral agents; elimination strategies.

\section{Introduction}

The hepatitis $\mathrm{C}$ virus (HCV) is one of the most common blood-borne pathogens in the world. An estimated 70 million persons are infected with chronic $\mathrm{HCV}$, and it remains a major cause of cirrhosis and hepatocellular carcinoma. Recent data demonstrated that 30 countries account for $80 \%$ of $\mathrm{HCV}$ infections worldwide. ${ }^{[1]} \mathrm{HCV}$ was first recognized as a potential infectious agent in 1951 when an outbreak of serum hepatitis was identified in a group of individuals who injected drugs, with other cohorts also subsequently reported in that decade. ${ }^{[2,3]}$ In 1989 , both a circulating antibody and a cDNA clone were identified in those with non-A non-B hepatitis, and it was officially named hepatitis C..$^{[4,5]}$ Since then, the identification of HCV genotypes, the demonstration of inter-

Received: April 13, 2020; Accepted: April 28, 2020; Available online: May 21, 2020 Corresponding author: Paul Y Kwo; Division of Hepatology, Department of Gastroenterology, Stanford University School of Medicine, 430 Broadway, Pavilion C, $3^{\text {rd }}$ Floor, Redwood City, CA 94063, USA

e-mail: pkwo@stanford.edu

(c) (i) (8) OPEN ACCESS

cc. This work is licensed under a Creative Commons Attribution-NonCommercial 4.0 International License.

(C) Copyright 2020 by Hepatology Forum - Available online at www.hepatologyforum.org feron and ribavirin as effective therapy for HCV, and finally, the introduction of direct-acting antiviral agent (DAAs) as treatment agents for $\mathrm{HCV}$, have revolutionized our ability to cure those with chronic HCV. Indeed, there are few diseases that have experienced such diagnostic and therapeutic advances over such a short period of time as have been observed with chronic HCV.

Highly effective DAAs for HCV are now available worldwide, including pan-genotypic (sofosbuvir/daclatasvir, sofosbuvir/velpatasvir, glecaprevir/pibrentasvir) therapies, as well as genotype-specific therapies. Regardless of the choice of treatment, successful completion of DAA therapy leads to sustained response rates of $90 \%$ or higher. Recent studies have also demonstrated improved survival and reduced liver-related complications in those with advanced liver disease and reduced rates of hepatocellular carcinoma. ${ }^{[6,7]}$ The cost of treating those with HCV with DAAs has been markedly reduced in both resource-rich and resource-poor countries since their introduction in 2014. One important intervention has been the introduction of low-cost generic DAAs that have been made available to countries that have limited resources to pay for HCV treatment; courses of therapy now cost less than US \$100 in many countries. ${ }^{[1]}$ As a result, many of the restrictions that had initially been placed on access to DAAs by some payers at the time of their introduction have gradually been lifted, including requirements related to an advanced fibrosis level, sobriety, and subspecialty care. Treatment is now widely available in many parts of the world.

With the availability of pan-genotypic therapy, the treatment of HCV has been simplified by eliminating much of the evaluation and monitoring that has historically been performed (https://www.hcvguidelines. org/treatment-naive/simplified-treatment). ${ }^{[8]}$ These advances have led national and international organizations to call for strategies to eliminate viral hepatitis (hepatitis $\mathrm{C}$ and hepatitis $\mathrm{B}$ ) as public health threats by 2030. Specifically, the World Health Organization has set targets that $90 \%$ of individuals with viral hepatitis could be diagnosed, and $80 \%$ of these individuals successfully treated, leading to a $65 \%$ reduction in mortality (https://www.who.int/hepatitis/publications/hep-elimination-by-2030-brief/en/). Currently, there are cities, states, countries, and other geographic units that are pursuing elimination strategies and setting ambitious targets to reduce and eliminate viral hepatitis as a public health threat.

When setting HCV elimination goals, it is important to accurately define what disease elimination means. Elimination, in general, can be defined as a reduction to zero incidence of infection in a geographical area while intervention measures are still required; an example of this is the elimination of poliomyelitis. ${ }^{[9]}$ Eradication implies permanent reduction to zero worldwide incidence and intervention measures are no longer required, with the eradication of smallpox being one example. 
Historically, the elimination or eradication of most infectious diseases has been the result of the development of effective vaccines to prevent the disease. Even with an effective vaccine, elimination and eradication can be difficult, as has been demonstrated with hepatitis $\mathrm{B}$, for which an effective vaccine is currently available. One exception has been the successful elimination of the infectious parasite onchocerciasis, which causes river blindness; over 100 million individuals have been treated, typically with ivermectin, and this disease has been eliminated in many countries. For HCV, elimination will likely be achieved using microelimination strategies in combination with broader strategies targeting those that are undiagnosed as well as at-risk populations.

The pathway to eliminate HCV involves multiple steps, including access to diagnostic testing, confirmation of active infection, referral for evaluation, disease staging, initiation of DAA therapy, and confirmation of cure. Worldwide, the largest gap in the cascade of care remains the challenge of the underdiagnosis of $\mathrm{HCV}^{\left[{ }^{[2]}\right.}$ It has been estimated that worldwide there are 70 million individuals infected with chronic $\mathrm{HCV}$ and that just $20 \%$ have been diagnosed, with an even smaller percentage (7\%) treated. Strategies to initiate widespread testing for HCV should help reduce this gap in diagnosis. One example is the recent recommendation by the United States Preventive Services Task Force to perform universal testing for HCV infection in patients 18 to 79 years of age (https://www.uspreventiveservicestaskforce.org/uspstf/recommendation/hepatitis-c-screening) rather than risk-based screening. Accompanying this challenge is how to confirm active viral replication. In many resource-constrained areas of the world, confirming active viral replication can be the most expensive and logistically challenging step once exposure to $\mathrm{HCV}$ has been confirmed. Point-of-care testing to confirm viremia through polymerase chain reaction techniques or the use of assays such as HCV core antigen testing will be required to allow elimination strategies to move forward. If this is not feasible, another option is to offer an inexpensive treatment (DAAs) to those who are anti-HCV antibody-positive even though some individuals who do not have active viremia will be exposed to DAAs. One could use the regional prevalence of active infection for those who are anti-HCV positive to help determine if this strategy might be effective: however, in some developed countries, the number of viremic patients is falling. ${ }^{[10]}$

Once the diagnosis of $\mathrm{HCV}$ infection is established, improved access to care will be essential to obtain DAA therapies. Historically, HCV has been treated by specialists, due to the complexities of monitoring those who were on interferon and ribavirin therapy. With the introduction of oral DAA therapy, the vast majority of the complexities of monitoring those on treatment are no longer present and oral DAA therapy can be safely given to those with compensated liver disease with little or no monitoring. ${ }^{[8]}$ Thus, our models of care are evolving from a centralized model of care, where patients seek out HCV evaluation and treatment with specialists, to a decentralized model of care with point-of-care testing wherever those with HCV infection are seeking care, including primary healthcare providers, clinics for those with drug and alcohol use disorders, sexual health clinics, needle exchange services, community health centers, and prisons, among others. The use of telehealth and extended community health outreach models have also been shown to be effective in teaching healthcare providers to treat patients with $\mathrm{HCV}$, including those with high-risk behaviors, in their local communities. ${ }^{[11]}$

As we evolve to include decentralized models of care for the treatment of $\mathrm{HCV}$, it is important to provide education worldwide about the importance of treatment so that those who are infected understand the necessity of obtaining therapy for HCV. This is especially important with DAA therapy, which is well-tolerated and is associated with sustained virologic response (SVR) rates that virtually guarantee cure. Even though cure rates are well above $90 \%$ for HVC, there will need to be additional investments worldwide in harm reduction services for comorbid conditions. Effective treatment of opioid addiction, directly observed therapies, and other strategies will be required in addition to universal access to DAAs. ${ }^{[12,13]}$

With more emphasis on decentralized care, the treatment of HCV will need to evolve from subspecialty care to community-based care. Multiple studies in the United States have demonstrated that sustained response rates are not compromised in any way when care is supervised by generalists, and similar results have been reported worldwide. ${ }^{[14]}$ In Egypt, an educate, test, and treat program was deployed to eliminate $\mathrm{HCV}$ infection in rural communities. In a population of rural villages consisting of 310,814 individuals, 33,839 (16.5\%) were HCV antibodypositive, 15,892 (47\%) had detectable HCV RNA, and 14,495 (91.2\%) initiated treatment. ${ }^{[7]}$ The treatment completion rate was $99.9 \%$, with 98.3\% of these treated individuals achieving SVR. Overall this decentralized model cured $84.6 \%$ of the estimated 17,137 infected persons across 73 villages in rural Egypt.

Other regions of the world have had similar success. India has released a national action plan to eliminate viral hepatitis and recently reported initial results from the Punjab model of HCV elimination using training models with e-learning and e-courses. ${ }^{[15]}$ In the preliminary report, 48,088 individuals were enrolled, assessed, and treated for HCV. Primary care providers from 3 university and 22 district hospitals were trained to provide algorithm-based DAA therapy using generic all-oral DAA regimens and were supervised by tele-health clinics using noninvasive measures used to assess fibrosis. The interim results demonstrated a per protocol SVR rate of $91.2 \%$ with a $67.6 \%$ intention-to-treat SVR rate. Those with cirrhosis and those with genotype 3 infection had comparable SVR rates to those without the predictors of poor response. Multiple other successful elimination efforts have been reported, including in the Republic of Georgia, Mongolia, and the Cherokee nation. ${ }^{[16-18]}$

These approaches can succeed among differing populations with varying comorbidities. The most important group of high-risk patients with $\mathrm{HCV}$ infection that must be engaged is intravenous drug users. This population worldwide may comprise 10 million individuals, with up to two-thirds infected with $\mathrm{HCV}^{\left[{ }^{[19]}\right.}$ These individuals may require more intensive care with enrollment in opiate substitution programs and other harm-reduction initiatives. One recent report demonstrated that high SVR rates can be achieved regardless of treatment approach: directly observed therapy, group therapy, or traditional individual therapy. ${ }^{[12]} \mathrm{Ef}-$ forts to engage high-risk populations with chronic $\mathrm{HCV}$ can reduce the incidence of chronic HCV. The recently reported Treatment as Prevention for Hepatitis C (TRAP Hep C) protocol from Iceland reported that $95 \%$ of eligible individuals were initiated on DAA therapy during the first 3 years of implementation and there was a marked reduction in the incidence of new HCV cases. ${ }^{[20]}$ While there are unique aspects to Iceland, including its small population and centralized care, this project demonstrates the value of the testing and treatment approach. Another population with recently reported success is the prison population, which has a high prevalence of $\mathrm{HCV}$ worldwide. A recent report from Australia has demonstrated that it is feasible to treat those who are incarcerated using decentralized care models and primary care providers. ${ }^{[21]}$

As we gain more experience with widespread diagnosis and treatment of HCV, a universal care pathway should be established that incorporates screening with the diagnostic testing and confirmation of active infection (Fig. 1) that will help move treatment from a specialistoriented centralized model to a decentralized model of care. Ideally, 


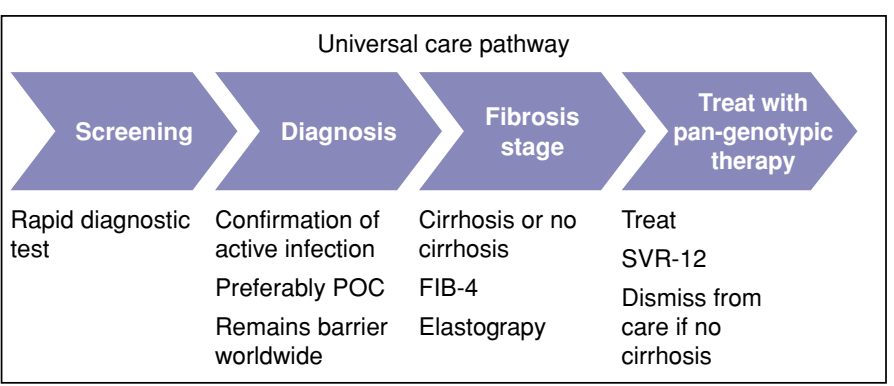

Figure 1. Universal care pathway for hepatitis $C$.

FIB-4: Fibrosis-4 Index for Liver Fibrosis; POC: Point-of-care; SVR-12: Sustained virologic response for 12 weeks.

these tests can be combined and should be point-of-care. Establishing a diagnosis of $\mathrm{HCV}$ remains the greatest barrier at this time to worldwide elimination efforts. Another important aspect of HCV elimination is the determination of hepatic fibrosis. Numerous noninvasive tests, including serum markers, platelet count, and fibrosis indices (aspartate aminotransferase-to-platelet ratio index and the fibrosis-4 index for liver fibrosis), as well as technologies such as elastography are available to help generalists assess the level of fibrosis or to determine if cirrhosis is present. ${ }^{[22]}$ Once sustained response has been confirmed, the vast majority of these individuals can be dismissed from care if no cirrhosis is present. Monitoring should be continued for those with cirrhosis and those with ongoing risk factors for reinfection. HCV elimination programs should be integrated into existing health systems, along with the appropriate funding to ensure the diagnosis, treatment, and monitoring of these individuals. ${ }^{[23]}$ Finally, the results of the many elimination programs should be reported such that best practices are available worldwide. Indeed, in the elimination of $\mathrm{HCV}$, it will take a village.

Peer-review: Externally peer-reviewed.

Potential Conflict of Interest: Paul Kwo

Advisory Board AbbVie, Arrowhead, Conatus, Durect Corp, Edigene, Eisai, Ferring, Gilead, Hep quant, Surrozen, Ribavirin Pregnancy Registry, Aligos, Mallinckrodt.

Research Grant: Allergan, Assembly, BMS, Gilead, Target Registries, Eiger, Arrowhead, Novartis

Stockholder: Durect

Other: Janssen (Data and Safety Monitoring Board).

\section{References}

1. Polaris Observatory HCV Collaborators. Global prevalence and genotype distribution of hepatitis $\mathrm{C}$ virus infection in 2015: a modelling study. Lancet Gastroenterol Hepatol 2017;2(3):161-176.

2. Appelbaum E, Kalkstein M. Artificial transmission of viral hepatitis among intravenous diacetylmorphine addicts. J Am Med Assoc 1951;147(3):222-224.

3. Alter AA, Michael M Jr. Serum hepatitis in a group of drug addicts. N Engl J Med 1958;259(8):387-389.

4. Kuo G, Choo QL, Alter HJ, Gitnick GL, Redeker AG, Purcell RH, et al. An assay for circulating antibodies to a major etiologic virus of human non-A, non-B hepatitis. Science 1989;244(4902):362-364.

5. Choo QL, Kuo G, Weiner AJ, Overby LR, Bradley DW, Houghton M. Isolation of a cDNA clone derived from a blood-borne non-A, non-B viral hepatitis genome. Science. 1989;244(4902):359-362.

6. Backus LI, Belperio PS, Shahoumian TA, Mole LA. Impact of Sustained
Virologic Response with Direct-Acting Antiviral Treatment on Mortality in Patients with Advanced Liver Disease. Hepatology 2019;69(2):487-497.

7. Backus LI, Belperio PS, Shahoumian TA, Mole LA. Direct-acting antiviral sustained virologic response: Impact on mortality in patients without advanced liver disease. Hepatology 2018;68(3):827-838.

8. Dieterich DT. A Simplified Algorithm for the Management of Hepatitis C Infection. Gastroenterol Hepatol (N Y) 2019;15(5 Suppl 3):1-12.

9. Dowdle WR. The principles of disease elimination and eradication. Bull World Health Organ 1998;76 (Suppl 2):22-25.

10. Zou B, Yeo YH, Le MH, Henry L, Chang ET, Lok AS, et al. Prevalence of Viremic Hepatitis C Virus Infection by Age, Race/Ethnicity, and Birthplace and Disease Awareness Among Viremic Persons in the United States, 19992016. J Infect Dis 2020;221(3):408-418.

11. Arora S, Kalishman S, Thornton K, Dion D, Murata G, Deming P, et al. Expanding access to hepatitis $\mathrm{C}$ virus treatment--Extension for Community Healthcare Outcomes (ECHO) project: disruptive innovation in specialty care. Hepatology 2010;52(3):1124-133.

12. Akiyama MJ, Norton BL, Arnsten JH, Agyemang L, Heo M, Litwin AH. Intensive Models of Hepatitis C Care for People Who Inject Drugs Receiving Opioid Agonist Therapy: A Randomized Controlled Trial. Ann Intern Med 2019;170(9):594-603.

13. Dore GJ, Altice F, Litwin AH, Dalgard O, Gane EJ, Shibolet O, et al; CEDGE CO-STAR Study Group. Elbasvir-Grazoprevir to Treat Hepatitis C Virus Infection in Persons Receiving Opioid Agonist Therapy: A Randomized Trial. Ann Intern Med 2016;165(9):625-634.

14. Beste LA, Glorioso TJ, Ho PM, Au DH, Kirsh SR, Todd-Stenberg J, et al. Telemedicine Specialty Support Promotes Hepatitis C Treatment by Primary Care Providers in the Department of Veterans Affairs. Am J Med 2017;130(4):432-438.e3.

15. Dhiman RK, Grover GS, Premkumar M, Taneja S, Duseja A, Arora S, et al; MMPHCRF Investigators. Decentralized care with generic direct-acting antivirals in the management of chronic hepatitis $\mathrm{C}$ in a public health care setting. J Hepatol 2019;71(6):1076-1085.

16. Tsertsvadze T, Gamkrelidze A, Chkhartishvili N, Abutidze A, Sharvadze L, Kerashvili V, et al. Three years of progress towards achieving hepatitis $\mathrm{C}$ elimination in the country of Georgia, April 2015 - March 2018. Clin Infect Dis 2019 Sep 29:ciz956.

17. Bat-Ulzii P, Lkhagva-Ochir O, Dashtseren B, Boldbaatar D, Davaadorj $\mathrm{Z}$, Ochirsum B, et al. Interim results of hepatitis prevention, control and elimination program in Ulaanbaatar, Mongolia. The International Liver Congress $^{\text {TM }} 2019$.

18. Mera J, Vellozzi C, Hariri S, Carabin H, Drevets DA, Miller A, et al. Identification and Clinical Management of Persons with Chronic Hepatitis C Virus Infection - Cherokee Nation, 2012-2015. MMWR Morb Mortal Wkly Rep 2016;65(18):461-466.

19. Grebely J, Dore GJ. Can hepatitis C virus infection be eradicated in people who inject drugs? Antiviral Res 2014;104:62-72.

20. Olafsson S, Fridriksdottir RH, Tyrfingsson T, Runarsdottir V, Hansdottir I, Bergmann OM, et al. Iceland may already have reached the WHO 2030 targets for diagnosis and treatment of hepatitis $\mathrm{C}$ virus infection: Results from the treatment as prevention for hepatitis $\mathrm{C}$ (Trap HepC) program. J Hepatol 2019;70:e337-e8.

21. Papaluca T, McDonald L, Craigie A, Gibson A, Desmond P, Wong D, et al. Outcomes of treatment for hepatitis $\mathrm{C}$ in prisoners using a nurse-led, statewide model of care. J Hepatol 2019;70(5):839-846.

22. Loomba R, Adams LA. Advances in non-invasive assessment of hepatic fibrosis. Gut 2020:gutjnl-2018-317593.

23. Ward JW, Hinman AR. What Is Needed to Eliminate Hepatitis B Virus and Hepatitis C Virus as Global Health Threats. Gastroenterology 2019;156(2):297-310. 